INVESTIGACIONES

\title{
Auto-regulación del aprendizaje y rendimiento académico
}

\author{
Self-regulated learning and academic achievement \\ Autoregulação da aprendizagem e desempenho acadêmico
}

\author{
Stella Vázquez z y Florencia Daura ${ }^{\mathrm{a}}$ \\ ${ }^{a}$ Centro de Investigaciones en Antropología Filosófica y Cultura (CIAFIC), Consejo Nacional de \\ Investigaciones Científicas y Técnicas (CONICET). Federico Lacroze 2100 (1426), Ciudad Autónoma de \\ Buenos Aires, Argentina. Correo electrónico: stellavazquez@gmail.com, flodaura@ hotmail.com. Telf.: +54 11 \\ 47760913
}

\section{RESUMEN}

Se presentan los resultados de una experiencia de intervención para promover el aprendizaje auto-regulado en alumnos de nivel medio. En una muestra compuesta por 120 alumnos se observó que las medias más bajas se registran en la auto-regulación meta-cognitiva, la regulación del esfuerzo, el manejo del tiempo y del ambiente de estudio y las estrategias profundas de elaboración y de pensamiento crítico. Estas variables correlacionan significativa y positivamente con el rendimiento académico, teniendo más peso la regulación del esfuerzo. Los resultados se interpretan en relación con los estilos de aprendizaje y con el tipo de evaluación usada por los docentes. Se hacen sugerencias pedagógicas que relacionan el aprendizaje auto-regulado con criterios de educación integral.

Palabras clave: estilos de aprendizaje, rendimiento académico, autorregulación, intervención pedagógica.

\begin{abstract}
An intervention to encourage self-regulated learning in secondary school students is described. The sample is made up of 120 students. The lowest means are for metacognitive self-regulation, effort regulation, study time and environment management, and knowledge development and critical thinking as deep-learning strategies. These variables, particularly effort regulation, correlate significantly and positively with academic achievement. The results are interpreted in relation to learning styles and the type of assessment used by teachers. Instructional suggestions are made linking self-regulated learning with integral education criteria.
\end{abstract}

Key words: learning styles, academic achievement, self-regulation, instructional intervention.

\section{RESUMO}

Apresentam-se os resultados de uma experiência de intervenção para promover a autoregulação da aprendizagem em estudantes do Ensino Médio. Em uma amostra de 120 estudantes observou-se que as médias mais baixas foram registradas na autoregulação metacognitiva, a regulação do esforço, a gestão de tempo e do ambiente de estudo e as estratégias profundas de elaboração e de pensamento crítico. Estas variáveis correlacionam-se significativa e positivamente com o desempenho acadêmico, pesando mais a regulamentação do esforço. Os resultados são interpretados em relação aos estilos de aprendizagem e ao tipo de avaliação utilizada pelos docentes. Sugerem-se ações pedagógicas que relacionam a aprendizagem autoregulada com critérios de educação integral.

Palavras chave: estilos de aprendizagem, rendimento escolar, autoregulação, intervenção pedagógica. 


\section{AUTO-REGULACIÓN DEL APRENDIZAJE Y RENDIMIENTO ACADÉMICO}

Se presentan los resultados de una experiencia de intervención para promover el aprendizaje auto-regulado en alumnos de nivel medio. Se ha elegido el marco teórico del llamado aprendizaje auto regulado no solo por su vigencia en la bibliografía científica, sino porque el mismo puede ser pensado como parte del proceso de educación integral, ya que en su concepto se tiene en cuenta gran parte de los elementos esenciales de este proceso.

Por otra parte, si bien existen numerosos trabajos sobre la relación entre las dos variables anunciadas en el título, los resultados de los mismos no llegan a reflejarse en las prácticas pedagógicas, al menos en el caso de las escuelas de nuestro país, en las que sigue siendo un tema de preocupación el nivel de aprendizaje de los alumnos y, de modo particular, las carencias referidas a las habilidades implicadas en el aprendizaje auto-regulado, que explicitamos en el punto siguiente.

\subsection{EL APRENDIZAJE AUTO REGULADO}

Con la aparición de la Psicología cognitiva se produce un importante giro en los planteos de la Psicología de la Educación, en un principio en los ámbitos de investigación y académicos, pero luego también en sus múltiples aplicaciones a la actividad del aula.

El aprendizaje autorregulado (AAR) es un concepto-sistema que refiere al manejo general de la propia conducta académica, a través de procesos interactivos entre distintos sistemas de control: atención, meta-cognición, motivación, emociones, acción y control volitivo (Boekaerts y Niemivirta, 2000; Boekaerts y Corno, 2005). Hay varios modelos de AAR con elementos comunes, pero con énfasis en distintos aspectos (Puustinen y Pulkkinen, 2001). Para este trabajo asumimos los modelos de Pintrich y de Zimmerman, que, a nuestro juicio, presentan de un modo orgánico los pasos que permiten diseñar una intervención pedagógica.

Paul Pintrich define el AAR como un proceso activo-constructivo por el que el alumno fija sus propias metas de aprendizaje, monitorea, regula y controla su adquisición de conocimientos, motivación y conductas, guiado por las metas y los rasgos contextuales del entorno (Pintrich, 2000; Pintrich y Zusho, 2002; Schunk, 2005). Es un proceso cíclico y abierto en el que se distinguen tres fases (Zimmerman, 2002): 1) Fase previa, en la que se establecen las metas y se planifica el curso de acción, de acuerdo con creencias, orientaciones motivacionales y expectativas específicas de resultados: 2) Fase de desempeño, en la que el sujeto se observa y controla mediante diferentes estrategias meta-cognitivas (auto-instrucciones, focalización de la atención, etc.) y volitivas (Kuhl, 1984, 1985); y 3) Fase de reflexión, en la que el sujeto evalúa los resultados obtenidos, juzga acerca de las causas de estos (atribuciones causales) y se suscitan en él las respuestas afectivas que condicionan las conductas ulteriores, dando lugar a que el ciclo se reinicie ratificando o rectificando metas, planes de acción y creencias motivacionales.

Zimmerman (2000) explicita que en la perspectiva socio-cognitiva, el AAR se considera un proceso interactivo que entraña no sólo habilidades de conducta para manejar las contingencias ambientales, sino también el conocimiento y un sentido de auto-eficacia (personal agency) para activar esas habilidades en contextos relevantes. La auto-regulación refiere a pensamientos, sentimientos y acciones auto-generadas que se planifican y adaptan para el logro de metas personales. 
La primacía conceptual y aún temporal que se da a las metas en el AAR, permite emplear estos modelos en el contexto de un proyecto de educación integral, en el que el saber académico, si bien es un valor en sí mismo, no es el último fin del proceso, sino que se ordena a favorecer la apropiación de los valores que éste vehiculiza y, de este modo, generar conductas con un grado creciente de auto determinación. Un proceso de auto-dirección es un proceso que, por su esencia, está orientado a fines. Los fines o metas no solo están al final del mismo, sino que son los criterios con que, a cada paso, se juzga si se está en el buen camino y también son el sostén de la acción, pues esta tiene sentido solo si la meta sigue presente y si conserva su valor, en cuyo caso es el núcleo de la motivación. Obsérvese que esto, dicho de manera sintética y obvia, se constituye en una clave pedagógica para el docente que quiere promover el AAR desde una concepción de educación integral, que se define, precisamente, como un proceso que promueve la conciencia y compromiso con los valores que conducen al logro de los fines últimos de la vida, que cada uno debe personalizar a través de la definición cada vez más neta de su concepción del mundo y de la vida. En este contexto, la auto-regulación de las conductas académicas es un medio que supone y, a la vez, promueve el ejercicio de la libertad.

Dentro de los procesos de autorregulación que configuran los patrones de aprendizaje adaptativo, se distingue procesos cognitivos y motivacionales. Entre los primeros, el establecimiento de metas y la planificación de la actividad, el uso de estrategias para supervisar el proceso, para corregirlo durante su desarrollo y evaluarlo al final de cada trayecto, el uso de estrategias de conocimiento básicas, de elaboración y de reorganización. La regulación motivacional puede referirse a procesos internos y también a elementos externos, por ejemplo, el manejo del ambiente para evitar distractores.

\section{HIPÓTESIS}

A partir de estos elementos teóricos, se plantea un trabajo de exploración e intervención, con las siguientes hipótesis:

1) Los alumnos llegan a la última etapa del nivel medio con deficiencias respecto de su capacidad de estudio autónomo, tanto por factores de índole motivacional, como por inadecuada formación de hábitos de auto-dominio y por falta de conocimiento de las estrategias específicas que permiten abordar con provecho las tareas académicas.

2) La calidad del rendimiento escolar se halla relacionado con la capacidad de auto-regulación.

\section{MÉTODO Y PROCEDIMIENTOS}

\subsection{MUESTRA}

Se trabajó con una muestra compuesta por 120 alumnos -65 varones y 55 mujeres, con una edad media de $15,6, \mathrm{SD}=.69$ - que cursan el ciclo de nivel medio en una escuela de nivel medio de gestión privada perteneciente a una Universidad de la Provincia de Buenos Aires, Argentina. La muestra es intencional y se trabajó con todos los alumnos de los cursos seleccionados. La investigación es de tipo descriptivo y correlacional. El 
diagnóstico se llevó a cabo en toda la muestra, en tanto que para la intervención se dividió la muestra en grupo control y grupo experimental. En esta etapa se usó un diseño no experimental -ex post facto-, porque no se manipularon las variables independientes, sino que se observaron en su contexto natural. En la intervención el diseño es de antes y después -pre-experimental-, porque se trabajó con grupo control.

\subsection{VARIABLES E INSTRUMENTOS DE MEDICIÓN}

La variable dependiente es el rendimiento escolar y la independiente, el nivel de posesión de los componentes del aprendizaje auto regulado. El rendimiento escolar se midió con las calificaciones de cada una de las materias en cada trimestre y con el promedio - de cada trimestre y final.

Las variables de auto-regulación se evaluaron con el MSLQ -Cuestionario de motivación y estrategias de aprendizaje (Pintrich y De Groot, 1990)-, un instrumento compuesto por 81 ítems distribuidos en 5 sub-escalas Lickert que evalúan los componentes cognitivos, meta-cognitivos y afectivos que forman parte del comportamiento académico auto-regulado, tal como son conceptualizados a partir del modelo motivacional de expectativa de logro por valor de la tarea (Eccles, 1983). Evalúa seis aspectos:

-Referidos al aspecto motivacional de metas, expectativas y valor:

- Metas: Intrínsecas y extrínsecas

- Auto-eficacia: creencia del sujeto en su capacidad de cumplir con éxito una tarea;

- Valor de la tarea: creencias acerca de la importancia intrínseca de una tarea y de su interés y utilidad para el sujeto;

- Ansiedad: la reacción afectiva negativa frente al aprendizaje en la escuela

-Referidos a aspectos cognitivos:

- uso de estrategias cognitivas: estrategias de repetición, elaboración, organización y pensamiento crítico.

- autorregulación: planificación, monitoreo de la propia actividad y manejo del esfuerzo.

Se administró en una hora de clase en el primer trimestre de 2009. Los puntajes de cada una de las sub-escalas se obtuvieron sumando las elecciones de los sujetos y convirtiendo la suma a escala 10 .

\subsection{PROCEDIMIENTOS DE PRUEBA DE LAS HIPÓTESIS}

La prueba de las hipótesis se hizo con metodología cuantitativa, que se complementó con análisis cualitativos, ya sea para ilustrar aspectos relevantes de los resultados, ya sea para presentar algunos de estos que no pueden abordarse de modo cuantitativo. Para el tratamiento de los datos recogidos se usó el Programa SPSS 11.5. La primera hipótesis requirió de análisis estadísticos descriptivos -media, mediana, desvío típico-, determinación de la normalidad -o no- de las distribuciones y decisión respecto del uso de estadísticos paramétricos o no paramétricos. Se hizo análisis correlacional -R de Pearson-, prueba $t$ de diferencia de medias y análisis de Cluster, que permitió detectar perfiles de grupos dentro de la muestra, los que fueron utilizados para el trabajo de intervención, en la medida en que permitieron tomar decisiones en cuanto a la planificación de las sesiones grupales y de las entrevistas individuales. Para la segunda hipótesis se usó el análisis correlacional y, a partir de allí, se decidió la posibilidad del análisis de regresión múltiple, tomando 
el rendimiento escolar como variable dependiente y las variables que correlacionan significativamente con éste como variables independientes (factores), a fin de determinar el tamaño del efecto en cada caso.

\section{LA INTERVENCIÓN PEDAGÓGICA}

La intervención referida a la mejora de los elementos de aprendizaje auto regulado se desarrolló en sesiones grupales de una hora reloj, con una frecuencia de dos veces por semana, a lo largo de 2 (dos) meses, durante el primer cuatrimestre del período lectivo 2009, complementadas con instancias de tutoría personal. Se utilizó como guía de trabajo el siguiente esquema temático:

1. ¿Qué es estudiar bien?:

- Reflexión sobre las metas: ¿Para qué estudiamos?

- Propuesta de valoración y de compromiso respecto de metas personales.

2. Organización y gestión del tiempo y ambiente de estudio: Toma de conciencia de las conductas habituales, análisis de su efectividad o pertinencia, enseñanza de estrategias de mejoramiento. ¿Cómo aprovechar las horas de clase?

- ¿Qué hacen los alumnos a los que les va bien?

- ¿Para qué y cómo tomar apuntes?

- Cómo controlar el propio proceso de comprensión (¿Estoy entendiendo? Preguntar, hacerse imágenes mentales, proponerse fijar lo que el profesor dice, relacionarlo con lo que ya se sabe sobre el tema).

- Usar estrategias de organización y de elaboración de los contenidos: Resumen, esquema, representación gráfica, de acuerdo con la diversa naturaleza de los contenidos.

3. El estudio después de clases:

- Planificar, establecer prioridades.

- Organizar los pasos del estudio o de la realización de trabajos.

- Hacer un cronograma, tratar de llevar una agenda.

- Controlar si tengo los apuntes completos, completar con el libro de texto, ampliar con fuentes de consulta (Biblioteca, Internet).

- Seleccionar y disponer el ambiente adecuado para el estudio o realización de tareas (tomar conciencia de los elementos distractores y prever cómo manejarlos).

- No posponer tareas (analizar cuándo y por qué hago esto).

- Saber pedir ayuda (profesores, compañeros, otros referentes académicos o del medio familiar o extra escolar).

\section{RESULTADOS DE LA FASE DIAGNÓSTICA}

\subsection{MOTIVACIÓN Y AUTO-REGULACIÓN}

Para evaluar los componentes que indican el perfil de auto-regulación cognitiva y motivacional de los alumnos, se aplicó el Cuestionario MSLQ (Pintrich y De Groot, 1990). 


\subsubsection{Confiabilidad del Instrumento}

Para el análisis de confiabilidad se usó el método de matriz de covarianza, obteniéndose valores de alfa de Cronbach satisfactorios para el cuestionario total y para cada una de las escalas:

Tabla 1. Confiabilidad del MSLQ

\begin{tabular}{|c|c|c|c|}
\hline Escala & alfa & Escala & alfa \\
\hline Total & .90 & Elaboración & .74 \\
\hline Metas Intrínsecas & .55 & Organización & .76 \\
\hline Metas Extrínseca & .61 & Pensamiento Crítico & .76 \\
\hline Valor tarea & .78 & Regulación meta-cognitiva & .80 \\
\hline Creencias de Control & .48 & Esfuerzo & .68 \\
\hline Auto-eficacia & .79 & Manejo de tiempo y ambiente & .66 \\
\hline Ansiedad & .59 & Pedir ayuda & .65 \\
\hline Repetición & .60 & Aprendizaje en grupo & .45 \\
\hline
\end{tabular}

\subsubsection{Validez del Instrumento}

Para verificar la validez de constructo se hicieron análisis factoriales. Los índices del Test de esfericidad de Bartlett (TEB), que son significativos en todos los casos, permiten rechazar la hipótesis nula de que la matriz muestral proviene de una población en que las variables no están correlacionadas. Los valores del KMO sustentan la hipótesis de que las variables pueden ser explicadas por un número menor de factores.

\subsubsection{Metas}

Se obtienen dos factores bien diferenciados $(\mathrm{KMO}=.72 ; \mathrm{X} 2(28)=128.36, \mathrm{p}<$ $.001 ; 48 \%$ de varianza explicada) que coinciden con los establecidos por los autores. En el primer factor cargan los ítemes correspondientes a metas extrínsecas, en el segundo los que definen metas intrínsecas, no hay ítemes complejos ni cargas negativas.

Tabla 2. Descriptivos y factores de metas

\begin{tabular}{|c|c|c|c|c|}
\hline Ítem & Media & SD & F1 & F2 \\
\hline Metas intrínsecas 1 & 1.94 & .77 & .14 & .67 \\
\hline Metas extrínsecas 7 & 2.18 & .92 & .66 & .00 \\
\hline Metas extrínsecas 11 & 1.90 & .95 & .70 & .20 \\
\hline Metas extrínsecas 13 & 1.51 & 1.1 & .56 & .28 \\
\hline Metas intrínsecas 16 & 2.46 & .86 & .16 & .70 \\
\hline Metas intrínsecas 22 & 1.77 & .93 & .29 & .40 \\
\hline Metas intrínsecas 24 & 1.12 & .82 & .06 & .67 \\
\hline Metas extrínsecas 30 & 2.03 & 1.0 & .72 & -.16 \\
\hline
\end{tabular}




\subsubsection{Valor de la tarea}

Se obtiene un solo factor $(\mathrm{KMO}=.77, \mathrm{X} 2(15)=184.28, \mathrm{p}<.001 ; 47 \%$ de varianza explicada), con cargas altas en todos los ítemes.

Tabla 3. Descriptivos y factor valor de la tarea

\begin{tabular}{|c|c|c|c|}
\hline Ítem & Media & SD & F1 \\
\hline 4 & 1.98 & .85 & .75 \\
\hline 10 & 1.84 & 1.02 & .74 \\
\hline 17 & 1.59 & .81 & .73 \\
\hline 23 & 1.53 & .79 & .71 \\
\hline 26 & 1.65 & .92 & .61 \\
\hline 27 & 1.83 & .94 & .56 \\
\hline
\end{tabular}

\subsubsection{Creencias de control de aprendizaje}

La variable creencias de control da por resultado dos factores $(\mathrm{KMO}=.53 ; \mathrm{X} 2$ $(6)=32.74, \mathrm{p}<.001 ; 66 \%$ de varianza explicada), lo que se justifica por el contenido de los ítems. En efecto, el primer factor contiene la atribución de buenos resultados al esfuerzo y la aplicación de estrategias adecuadas ${ }^{1}$ y el segundo factor, la atribución de malos resultados a falta de esfuerzo. ${ }^{2}$

Tabla 4. Descriptivos y factores de creencias de control

\begin{tabular}{|c|c|c|c|c|}
\hline Ítem & Media & SD & F1 & F2 \\
\hline 2 & 2.56 & .71 & .85 & .05 \\
\hline 18 & 1.53 & .98 & .79 & .11 \\
\hline 25 & 2.56 & .64 & -.07 & .85 \\
\hline 9 & 1.56 & .96 & .24 & .71 \\
\hline
\end{tabular}

\subsubsection{Auto-eficacia y ansiedad}

En consonancia con la teoría, hay dos factores $(\mathrm{KMO}=.765 ; \mathrm{X} 2(78)=184.28, \mathrm{p}$ $<.001 ; 43 \%$ de varianza explicada). Es interesante señalar que en algunos de los ítems hay oposición, es decir, que la ansiedad y la convicción de que se es eficaz se oponen, lo que resulta lógico. La auto-eficacia se define como la convicción de que se es capaz de logros; pero si se examina el modo de formulación de los ítems, se advierte que

1 02. Si estudio de modo correcto voy a ser capaz de aprender los temas de este año. 18. Si me esfuerzo voy a entender los temas de este año.

2 09. Si no aprendo lo que se enseña este año, es por mi culpa. 25. Si no entiendo lo que se desarrolla en clase es porque no me esfuerzo lo suficiente. 
en algunos ${ }^{3}$ se podría interpretar como una esperanza o expectativa, más que como un convencimiento. Los ítems más definidos ${ }^{4}$ son los que tienen la media más baja, lo que significa que los sujetos tienen más expectativas que seguridad en sus posibilidades.

Tabla 5. Descriptivos y factores de Autoeficacia y Ansiedad

\begin{tabular}{|c|c|c|c|c|}
\hline Ítem & Media & SD & F1 & F2 \\
\hline Ansiedad 3 & .86 & .94 & -.50 & .35 \\
\hline Auto-eficacia 5 & 1.43 & .77 & .66 & -.14 \\
\hline Auto-eficacia 6 & 1.90 & .92 & .58 & -.07 \\
\hline Ansiedad 8 & 1.00 & .94 & -.36 & .44 \\
\hline Auto-eficacia 12 & 2.44 & .69 & .61 & .02 \\
\hline Ansiedad 14 & 1.58 & 1.06 & -.07 & .55 \\
\hline Auto-eficacia 15 & 1.71 & .90 & .61 & -.30 \\
\hline Ansiedad 19 & .73 & .91 & -.30 & .72 \\
\hline Auto-eficacia 20 & 1.80 & .71 & .73 & .08 \\
\hline Auto-eficacia 21 & 2.48 & .639 & .49 & .326 \\
\hline Ansiedad 28 & 1.09 & .987 & .148 & .730 \\
\hline Auto-eficacia 29 & 1.91 & .797 & .65 & -.360 \\
\hline Auto-eficacia 31 & 1.91 & .722 & .72 & -.016 \\
\hline
\end{tabular}

\subsubsection{Estrategias cognitivas}

Se obtienen tres factores $(\mathrm{KMO}=.80 ; \mathrm{X} 2(171)=767.03, \mathrm{p}<.001 ; 50 \%$ de varianza explicada), el primero reúne los ítemes de pensamiento crítico con los de elaboración, excepto uno; el segundo corresponde a la estrategia de organización y carga también en este factor el ítem $67 ;^{5}$ el tercer factor agrupa los ítemes de la estrategia de repetición.

Tabla 6. Descriptivos y factores de estrategias cognitivas

\begin{tabular}{|c|c|c|c|c|c|}
\hline Ítem & Media & SD & F1 & F2 & F3 \\
\hline Organización 32 & 2.17 & 1.10 & -.07 & .60 & .25 \\
\hline Repetición 39 & 2.03 & 1.01 & -.13 & .02 & .77 \\
\hline Organización 42 & 2.30 & .82 & .28 & .63 & .21 \\
\hline Pensamiento crítico 47 & 1.32 & .98 & .65 & .20 & -.07 \\
\hline
\end{tabular}

3 12. Tengo confianza en que puedo aprender los conceptos básicos de este año. 21. Espero desempeñarme bien este año.

4 05. Creo que voy a sacar excelentes notas este año. 15. Me siento seguro de poder entender las cosas más difíciles que enseñen los profesores este año.

5 67. Para estudiar me hago resúmenes cortos con las ideas principales que saco de mis apuntes, del libro, etc. 


\begin{tabular}{|c|c|c|c|c|c|}
\hline Organización 49 & 1.50 & 1.22 & .08 & .78 & .09 \\
\hline Pensamiento crítico 51 & 1.35 & .96 & .57 & .13 & .01 \\
\hline Organización 63 & 1.90 & 1.04 & .16 & .80 & .14 \\
\hline Repetición 59 & 1.98 & 1.02 & .25 & .17 & .51 \\
\hline Elaboración 62 & .99 & .95 & .65 & .03 & .21 \\
\hline Elaboración 64 & 1.56 & 1.02 & .71 & -.11 & .21 \\
\hline Pensamiento crítico 66 & 1.73 & .94 & .71 & .17 & -.08 \\
\hline Elaboración 67 & 2.09 & .99 & .12 & .75 & .05 \\
\hline Elaboración 69 & 1.75 & .87 & .70 & .13 & .23 \\
\hline Pensamiento crítico 71 & 1.43 & .92 & .66 & .19 & -.17 \\
\hline Repetición 72 & 1.35 & 1.00 & .15 & .30 & .47 \\
\hline Elaboración 81 & 1.56 & .95 & .75 & -.02 & .08 \\
\hline Pensamiento crítico 38 & 1.03 & .93 & .59 & .03 & .01 \\
\hline Repetición 46 & 1.76 & 1.00 & .02 & .23 & .68 \\
\hline Elaboración 53 & 1.63 & 1.03 & .43 & .26 & .21 \\
\hline
\end{tabular}

\subsubsection{Regulación meta-cognitiva}

Hay un solo factor $(\mathrm{KMO}=.754, \mathrm{X} 2(66)=360.21, \mathrm{p}<.001 ; 32 \%$ de varianza explicada) y los ítemes $55^{6}$ y $56^{7}$ son los que tienen la media más baja.

Tabla 7. Descriptivos y factores de regulación meta-cognitiva

\begin{tabular}{|c|c|c|c|}
\hline Ítem & Media & SD & F1 \\
\hline 33 & 1.39 & 1.05 & .53 \\
\hline 36 & 1.60 & 1.08 & .56 \\
\hline 41 & 2.48 & .68 & .55 \\
\hline 44 & 1.48 & .97 & .44 \\
\hline 54 & 1.57 & 1.07 & .55 \\
\hline 55 & 1.71 & 1.03 & .62 \\
\hline 56 & 1.29 & 1.08 & .42 \\
\hline 57 & 1.65 & .90 & .44 \\
\hline 61 & 1.50 & .96 & .55 \\
\hline 76 & 2.15 & .77 & .71 \\
\hline 78 & 1.72 & .96 & .65 \\
\hline 79 & 2.03 & .89 & .68 \\
\hline
\end{tabular}

55. Me hago preguntas para asegurarme de haber comprendido lo que estuve estudiando.

56. Trato de cambiar el modo de estudio para acomodarme a las exigencias de la materia y al estilo del profesor. 


\subsubsection{Manejo de tiempo y de ambiente}

De acuerdo con el análisis factorial, fue preciso sacar los ítemes 52,73 y $80 ;{ }^{8}$ el resto de los ítemes constituyen un solo factor $(\mathrm{KMO}=.77, \mathrm{X} 2(10)=114.36 \mathrm{p}<.001$; $52 \%$ de varianza explicada).

Tabla 8. Descriptivos y factores de manejo de tiempo y ambiente

\begin{tabular}{|c|c|c|c|}
\hline Ítem & Media & SD & F1 \\
\hline 35 & 1.66 & 1.10 & .74 \\
\hline 43 & 1.51 & 1.07 & .76 \\
\hline 65 & 1.88 & 1.13 & .63 \\
\hline 70 & 2.03 & .99 & .71 \\
\hline 77 & 1.12 & .99 & .65 \\
\hline
\end{tabular}

\subsubsection{Esfuerzo}

Se obtiene un único factor $(\mathrm{KMO}=.65 ; \mathrm{X} 2(6)=83.16, \mathrm{p}<.001 ; 52 \%$ de varianza explicada). Las medias más altas corresponden a los ítemes 74 y $60,{ }^{9}$ es decir, que los alumnos se auto-perciben como capaces de perseverar en el estudio.

Tabla 9. Descriptivos y factores de regulación del esfuerzo

\begin{tabular}{|c|c|c|c|}
\hline Ítem & Media & SD & F1 \\
\hline 37 & 1.13 & .95 & .71 \\
\hline 48 & 1.51 & .88 & .58 \\
\hline 60 & 1.88 & .97 & .72 \\
\hline 74 & 2.76 & 3.05 & .84 \\
\hline
\end{tabular}

\subsubsection{Aprendizaje en grupo y búsqueda de ayuda}

Las dos variables del instrumento original, en nuestra muestra forman un solo factor $(\mathrm{KMO}=.71 ; \mathrm{X} 2(15)=114.3, \mathrm{p}<.001)$. Las medias más altas corresponden a los ítemes de búsqueda de ayuda, la más baja al ítem $50,{ }^{10}$ porque los alumnos de nivel medio no están habituados a discutir los temas escolares: En este sentido, el ítem es más afín a la escala de pensamiento crítico.

8 52. Me resulta difícil seguir un plan para usar el tiempo de estudio. 73. Cumplo con la asistencia a clases. 80. Raramente me hago el tiempo para repasar las guías de clase y apuntes, antes de un examen.

9 60. Si lo que hay que hacer para una materia es difícil, o bien lo dejo o sólo estudio las partes fáciles. 74. Aunque lo que tenga que estudiar sea aburrido, me las arreglo para llegar hasta el final.

10 Cuando estudio, me reservo un tiempo para discutir los temas con un grupo de compañeros. 
Tabla 10. Descriptivos y factores de aprendizaje en grupo y búsqueda de ayuda

\begin{tabular}{|c|c|c|c|}
\hline Ítem & Media & SD & F1 \\
\hline Grupo 45 & 1.65 & .96 & .63 \\
\hline Grupo 50 & 1.32 & 1.00 & .60 \\
\hline Grupo 34 & 1.76 & 1.1 & .42 \\
\hline Ayuda 40 & 1.73 & 1.10 & .57 \\
\hline Ayuda 58 & 1.92 & .92 & .45 \\
\hline Ayuda 68 & 2.00 & .93 & .83 \\
\hline Ayuda 75 & 2.09 & .96 & .78 \\
\hline
\end{tabular}

\subsection{PERFIL MOTIVACIONAL Y DE AUTO-REGULACIÓN DE LA MUESTRA}

Para determinar el perfil motivacional de los alumnos, se hizo la media de las subescalas re-estructuradas a partir del análisis factorial y, luego, un análisis de Cluster.

Tabla 11. Descriptivos de las sub-escalas del MSLQ

\begin{tabular}{|c|c|c|}
\hline Variables & Media & SD \\
\hline Metas intrínsecas & 6.02 & 1.87 \\
\hline Metas extrínsecas & 6.34 & 2.24 \\
\hline Valor tarea & 5.62 & 1.77 \\
\hline Control del aprendizaje & 6.84 & 1.71 \\
\hline Autoeficacia & 6.50 & 1.64 \\
\hline Ansiedad & 3.50 & 1.98 \\
\hline Repetir & 5.96 & 2.26 \\
\hline Elaborar & 5.31 & 2.13 \\
\hline Organizar & 6.58 & 2.67 \\
\hline Pensamiento crítico & 4.59 & 2.24 \\
\hline Auto-regulación meta-cognitiva & 5.74 & 2.3 \\
\hline Tiempo y ambiente & 5.54 & 2.30 \\
\hline Regulación del esfuerzo & 5.70 & 2.14 \\
\hline Aprendizaje en grupo & 5.24 & 2.28 \\
\hline Pedir ayuda & 6.41 & 2.28 \\
\hline
\end{tabular}

No hay diferencias significativas entre las medias de ambos tipos de metas, que correlacionan moderadamente $(\mathrm{r}=.32, \mathrm{p}<.001)$, es decir, que los alumnos no las conciben como metas opuestas. En el caso de la motivación intrínseca, la media más alta corresponde al ítem 16 y la media más baja al ítem 24, lo que ilustra la combinación que 
hacen los alumnos entre ambos tipos de metas. Para la motivación extrínseca las medias más altas corresponden a los ítemes 7 y 30:11 El 80\% de los alumnos considera como lo más satisfactorio obtener notas altas y el $70 \%$ afirma que hace las cosas bien para demostrar a su familia y a los demás su capacidad; ambas metas son características de perfiles competitivos, que no son compatibles con el interés intrínseco y que son rasgos poco deseables desde la perspectiva de la formación de la personalidad. Por otra parte, cabe reconocer que los chicos viven en un mundo que valoriza e impulsa ese rasgo.

La motivación por el valor de la tarea tiene una de las medias más bajas, lo que parecería contradictorio con las metas intrínsecas. El ítem que tiene la media más alta dice: Para mí es importante aprender lo que se enseña en este año. En cambio, las medias más bajas corresponden a: Estoy muy interesado en los temas de las materias de este año y Me gustan los temas de las materias de este año. Es decir que los alumnos asignan importancia a aprender, pero no están motivados intrínsecamente por los contenidos, lo que confirma la interpretación hecha más arriba. Sin duda, esto es un indicador importante para el educador, respecto de la necesidad de mostrar el valor en sí y no solo instrumental de lo que se propone en el aula.

La variable creencias de control del aprendizaje es una forma de juicio de atribución de los resultados escolares al esfuerzo y, como observamos en el análisis precedente, encierra dos factores: el primero de ellos es el de atribución del éxito en el aprendizaje al propio esfuerzo, en tanto que el segundo es la atribución del fracaso a la falta de esfuerzo. Estos factores no correlacionan en la muestra, aparecen como independientes, con una significativa diferencia en las medias -8.53 y 3.80 , respectivamente-, lo que indica un sesgo en la atribución que es propio de patrones defensivos: Si me va bien es porque me esfuerzo, si me va mal no lo asumo como responsabilidad propia. Es interesante señalar que un análisis de varianza por sexos en estas variables, muestra diferencias significativas $(\mathrm{F}(1)=21.87, \mathrm{p}<.001)$ en la atribución del éxito al esfuerzo a favor de las mujeres (en cambio, en la atribución del fracaso no hay diferencias significativas). Para interpretar este resultado, podemos referir a estudios similares que indican que los varones tienen un patrón defensivo, por el cual tienden a atribuir el éxito más a la habilidad que al esfuerzo, en la creencia de que esto protege su auto-imagen: Si se reconoce el esfuerzo, parecería que se deteriora el concepto respecto de la capacidad. En este estilo atribucional, la inversión de esfuerzo suele ser visto como un indicador de falta de habilidad y, por lo tanto, amenaza la auto-valía: si las personas son capaces, no tienen que esforzarse tanto (Seifert, 1997, 2004), razín por la que los sujetos suelen usar la estrategia de retirar el esfuerzo para proteger su imagen. En el patrón defensivo también se atribuye el fracaso a factores externos, lo que, junto con la estrategia de sub-desempeño, es un modo de protección del self. A la vez, cabe tener en cuenta que las metas de tarea y el aprendizaje cooperativo reducen las estrategias auto-defensivas (Martin, Marsh y Debus, 2001a, 2001b). En cambio, en las mujeres hay una tendencia al patrón de indefensión -atribuir el fracaso a la falta de habilidad y el éxito a factores externos-, junto con una mayor valoración del esfuerzo.

11 16. En las materias de este año prefiero los temas que despiertan mi curiosidad, aunque sean difíciles de aprender. 24. Cuando tengo oportunidad en las materias de este año. Elijo tareas y ejercicios de los que pueda aprender mucho, aunque eso no me garantice una nota alta. 7. Este año, sacar una nota alta es lo más satisfactorio para mí. 30. Quiero hacer las cosas bien este año, para demostrar mi capacidad a mi familia y a los demás. 
En apoyo de esta interpretación está el hecho de que los varones tiene una media más alta en el ítem 25: Si no entiendo lo que se desarrolla en clase es porque no me esfuerzo lo suficiente, donde lo implícito es: Y no porque no soy capaz.

La auto-eficacia es un concepto introducido por Bandura (1977, 1993, 1995, 1997), en el contexto de las teorías socio-cognitivas de la motivación. Se la define como la auto-percepción y juicio acerca de la posibilidad de obtener logros, la convicción de que uno puede actuar adecuadamente en una situación, la creencia de la persona en su habilidad para llevar a cabo o afrontar con éxito una tarea específica. Sin duda el juicio de auto-eficacia se genera a partir del éxito en determinadas tareas, lo que genera confianza, convicción de competencia personal; en cambio, los fracasos generan convicción de falta de eficacia. En el juicio de auto-eficacia se distingue las dimensiones de magnitud, intensidad y generalidad. Esta última refiere a la discusión acerca de si el juicio de auto-eficacia se extiende a todas las actividades o si está ligado a ámbitos específicos, lo que parecería más lógico; aunque también podría pensarse que el sujeto extiende esa convicción a partir de experiencias específicas. Este juicio condiciona el grado de compromiso en distintas actividades, la persistencia en las mismas, el esfuerzo que se está dispuesto a invertir en ellas; por lo tanto, es evidente su rol en los procesos de aprendizaje y su relación con las dimensiones motivacionales descriptas más arriba.

En nuestra muestra, la creencia en la auto-eficacia tiene una media moderadamente alta y, como era de esperar, correlaciona positivamente con otra variables motivacionales:

Tabla 12. Correlaciones entre variables motivacionales y auto-eficacia

\begin{tabular}{|c|c|}
\hline Variables motivacionales & $\begin{array}{l}\text { Auto- } \\
\text { eficacia }\end{array}$ \\
\hline Metas intrínsecas & $.55(* *)$ \\
\hline Metas extrínsecas & $.30(* *)$ \\
\hline Valor de la tarea & $.50(* *)$ \\
\hline Atribución del éxito al esfuerzo & $.28(* *)$ \\
\hline Atribución del fracaso & -.18 \\
\hline Ansiedad & $-.28(* *)$ \\
\hline Auto-regulación & $.57(* *)$ \\
\hline
\end{tabular}

La auto-regulación meta-cognitiva es la capacidad de advertir y de controlar los procesos mediante los cuales se aprende. El primer aspecto comprende estrategias referidas al qué, cómo y cuándo; es decir, al conocimiento declarativo-qué es lo que sé, qué es lo que no tengo claro-, al conocimiento procedimental -cuál es la forma más eficaz de abordar un contenido, qué estrategias usar en cada situación- y al conocimiento condicional -cuándo emplear diversas estrategias. El segundo aspecto es el más propiamente regulativo, porque incluye estrategias de guía y de corrección de los propios procesos, tales como volver a leer cuando advierto que no estoy entendiendo, hacerme preguntas para cerciorarme del nivel de comprensión, etc. 
En nuestra muestra se registra una media de auto-regulación relativamente baja.

Las estrategias de regulación meta-cognitiva más usadas son las de volver atrás en la lectura cuando algo no se comprende bien, determinar qué conceptos no se comprenden y aclarar las dudas que surgen durante la clase (más del $80 \%$ de los alumnos informan el uso de estas estrategias). En cambio, las menos frecuentes son las auto-preguntas y la flexibilidad en el modo de estudio (más del $60 \%$ no emplean nunca o casi nunca estas estrategias). Por otra parte, un $48 \%$ de los alumnos informa que con frecuencia se distrae durante las clases y pierde lo importante.

En el uso de las estrategias cognitivas, los alumnos reconocen sus carencias. Las medias más altas corresponden a las estrategias básicas del subrayado y la búsqueda de ideas principales (las usan siempre o casi siempre entre el $75 \%$ y $80 \%$ de los alumnos), que, muy probablemente, han ejercitado desde la escuela primaria. En cambio, los ítemes con la media más baja se refieren a estrategias más complejas, tales como el de buscar relaciones entre contenidos de distintas materias -estrategia que apunta a gestar un hábito de cosmovisión, empleada sólo por un $27 \%$ de los alumnos-, analizar los argumentos que sostienen lo que se les enseña en clase o leen en el libro -llamadas estrategias de pensamiento crítico, pero que en realidad son hábitos de búsqueda de razones-, que el $70 \%$ no emplea casi nunca. Finalmente, también son deficitarias las estrategias básicas de repetición (el 57\% no recurre nunca a ellas), que son imprescindibles para fijar contenidos conceptuales y disponer así de una mayor memoria de trabajo. Cabe señalar que los alumnos arrastran estos hábitos y carencias hasta los estudios superiores, confundiendo el acto de estudio con el de lectura y, a lo sumo, el subrayado.

Otra variable clave es el manejo del tiempo y ambiente de estudio que hacen los alumnos. La media en este aspecto es una de las más bajas -5.13- y sólo un $20 \%$ de los alumnos supera los 7 puntos. De acuerdo con el auto-informe, la estrategia más usada es la de buscar un lugar de estudio que favorezca la concentración -el 80\% de los alumnos lo hacen siempre o casi siempre-, aunque no tengan un lugar fijo; pero la mayoría $-65 \%$ - reconoce que no usa bien el tiempo de estudio y un $40 \%$ reconoce que no tiene estrategias para asegurarse de completar tareas y lecturas de cada materia. Estos resultados deben interpretarse en relación con el interés por las tareas que propone la escuela y con el esfuerzo que están dispuestos a invertir en las tareas escolares.

Dado que se observan correlaciones significativas entre los distintos aspectos explorados, referidos a estrategias y a motivación, se hace un análisis de Cluster.

En nuestra muestra hemos obtenido tres grupos (Ver Tabla 13) que se diferencian significativamente entre sí y que permitirían reconocer tres estilos de aprendizaje, que hemos llamado: superficial, pragmático y profundo.

Tabla 13. Medias de estrategias, motivación y regulación por estilos de aprendizaje

\begin{tabular}{|c|c|c|c|}
\hline & Superficial & Pragmático & Profundo \\
\hline Metas intrínsecas & 4.23 & 5.88 & 6.69 \\
\hline Metas extrínsecas & 4.90 & 6.11 & 6.90 \\
\hline Valor de la tarea & 3.87 & 5.71 & 6.22 \\
\hline Autoeficacia & 4.95 & 7.62 & 6.77 \\
\hline Ansiedad & 3.79 & 2.67 & 3.60 \\
\hline
\end{tabular}




\begin{tabular}{|c|c|c|c|}
\hline Repetir & 4.13 & 5.69 & 6.67 \\
\hline Elaborar & 3.01 & 5.03 & 6.19 \\
\hline Organizar & 3.40 & 6.62 & 7.69 \\
\hline Pensamiento Crítico & 2.64 & 5.56 & 5.04 \\
\hline Auto-regulación meta-cognitiva & 3.84 & 5.49 & 6.47 \\
\hline Manejo tiempo y ambiente & 4.39 & 4.54 & 6.18 \\
\hline Esfuerzo & 3.56 & 5.65 & 6.46 \\
\hline Aprender en grupo & 4.32 & 2.34 & 6.26 \\
\hline Pedir ayuda & 5.42 & 3.47 & 7.47 \\
\hline
\end{tabular}

1. Superficial: Este grupo, de 26 alumnos, se caracteriza por el bajo empleo de estrategias cognitivas, muy poca capacidad de auto-regulación y de motivación intrínseca. Es el grupo de menor rendimiento académico.

2. Pragmático: Es un grupo de18 alumnos en el que prima la motivación extrínseca y el valor instrumental de las tareas escolares y la creencia de auto-eficacia; usan estrategias, principalmente la de organización, el manejo del tiempo y del esfuerzo, que es moderado; no se inclinan por el aprendizaje grupal ni son proclives al pedido de ayuda. Tienen menor nivel de ansiedad que los otros dos grupos. Es el grupo con rendimiento más alto.

3. Profundo: Este grupo de 74 alumnos, que llamamos de estilo profundo, sobresale por sus metas y el valor que le da a la tarea escolar, informan del uso de estrategias básicas y de organización, manejan su tiempo, ambiente y esfuerzo, buscan ayuda y prefieren el aprendizaje en grupo. Tienen un nivel medio de ansiedad. Es el grupo que más se dedica a las tareas escolares, con un rendimiento algo más bajo que el del grupo pragmático.

Figura 1. Estilos de aprendizaje

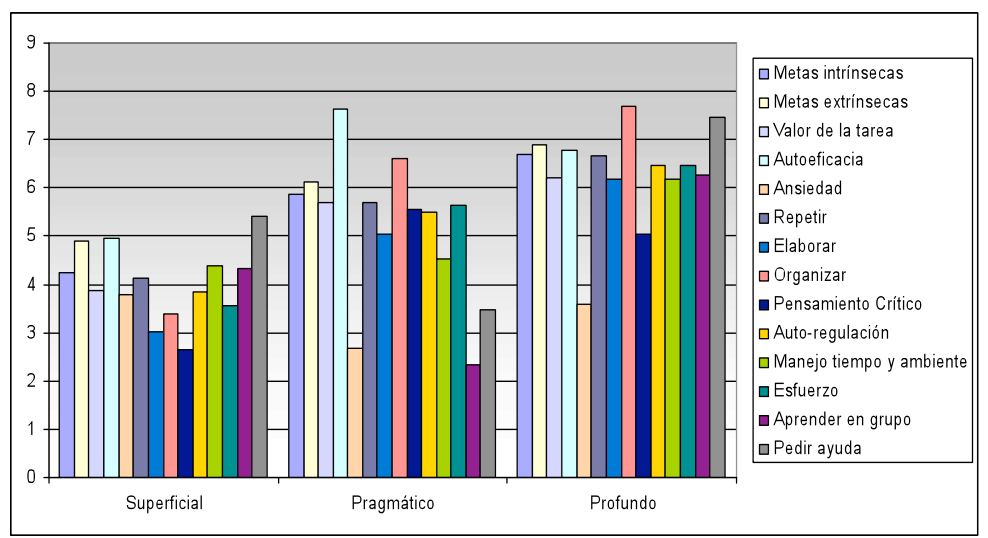




\subsection{EL RENDIMIENTO ACADÉMICO}

Este aspecto fue evaluado a través de las calificaciones en cada materia y trimestre; se calculó, además, el promedio para cada trimestre. Para evaluar la relación entre el rendimiento y los componentes del aprendizaje auto-regulado se hizo un análisis correlacional y, sobre esa base, un análisis de regresión múltiple, tomando como variable dependiente el rendimiento final.

Tabla 14. Correlaciones entre rendimiento y componentes del SRL

\begin{tabular}{|c|c|c|c|}
\hline & 1er. Trim. & 2do. Trim. & 3er. Trim. \\
\hline Promedio 1er. trimestre & & .68 & .86 \\
\hline Promedio 2do. trimestre & .68 & & .72 \\
\hline Promedio 3er. trimestre & .86 & .72 & \\
\hline Metas intrínsecas & .30 & .30 & .30 \\
\hline Metas extrínsecas & -.06 & .03 & -.03 \\
\hline Valor de la tarea & .13 & .10 & .19 \\
\hline Autoeficacia & .35 & .30 & .46 \\
\hline Ansiedad & -.33 & -.30 & -.30 \\
\hline Repetir & -.05 & .08 & -.04 \\
\hline Elaborar & .18 & .22 & .30 \\
\hline Organizar & .21 & .30 & .30 \\
\hline Pensamiento crítico & .30 & .19 & .30 \\
\hline Auto-regulación meta-cognitiva & .35 & .30 & .36 \\
\hline Manejo de tiempo y ambiente & .32 & .30 & .38 \\
\hline Regulación del esfuerzo & .42 & .41 & .48 \\
\hline Aprendizaje en grupo & .12 & .04 & .11 \\
\hline Pedir ayuda & .06 & .15 & .14 \\
\hline
\end{tabular}

El rendimiento académico correlaciona positiva y significativamente con la regulación del esfuerzo, el manejo de tiempo y ambiente, la creencia de Autoeficacia y en menor grado con la auto-regulación meta-cognitiva, las estrategias cognitivas de pensamiento crítico y de elaboración y las metas intrínsecas. Sin embargo, en el análisis de regresión $(\mathrm{F}=(3) 17.47$, y $115, \mathrm{R} 2=0.33, \mathrm{p}<.001)$, las únicas variables predictoras son el esfuerzo, la auto-eficacia y el manejo de tiempo y de ambiente, que en conjunto permiten predecir el 33\% de la varianza en el rendimiento académico. Este resultado es un tanto llamativo, pues podría interpretarse en el sentido de que en el rendimiento no influyen las estrategias cognitivas ni las metas intrínsecas; sin embargo, si se tiene en cuenta el modo en que se operativizó el rendimiento, nuestra interpretación se desplaza hacia el modo de evaluar de los docentes, que reflejaría una carencia en cuanto a la consideración de los procesos que influyen en la calidad de los aprendizajes y un acento puesto en los resultados. En el caso de la auto-eficacia, su peso se explica porque esta creencia 
se genera por las experiencias previas: Los alumnos que se han adecuado al modo de evaluar de los docentes logran calificaciones más altas y desarrollan la creencia de que son capaces de obtener buenos resultados.

\subsection{EL RENDIMIENTO ACADÉMICO Y LOS ESTILOS DE APRENDIZAJE}

Un análisis de varianza muestra que hay relaciones significativas $(\mathrm{F}=(114) 11.85$, $\mathrm{p}<.001)$ entre ambas variables, en cuanto el estilo superficial ha tenido el rendimiento más bajo a lo largo del año y los estilos pragmático y profundo el rendimiento más alto (Ver Figura 2).

Figura 2. Medias de rendimiento académico por estilos de aprendizaje

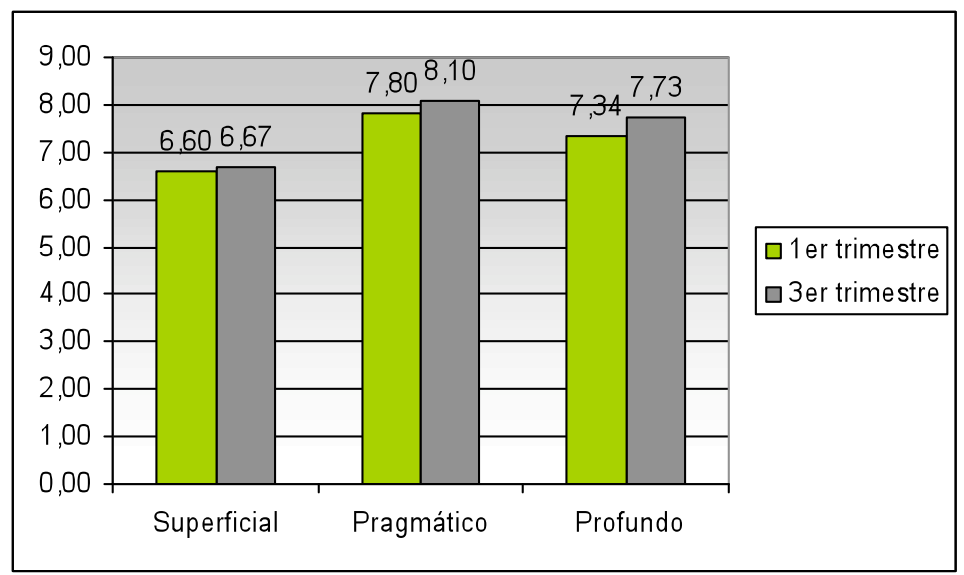

\section{RESULTADOS DE LA INTERVENCIÓN}

La intervención orientada a promover los distintos aspectos de la auto-regulación se desarrolló a través de acciones de tutoría grupal y tutoría personal.

La tutoría grupal se realizó en sesiones de 40 a 80 minutos cada semana durante dos meses. En el primer encuentro se explicaron algunos de los aspectos que evalúa el cuestionario MSLQ, a fin de que el alumnado comprenda las variables en juego y sea capaz de predecir los resultados de la aplicación. Así, se los invitó en cada sesión grupal a identificar a los compañeros de curso que más probablemente obtuvieron el mejor puntaje en la medición de la variable presentada por el docente a cargo. Esta estrategia provocó una mejor participación de todo el alumnado y, a su vez, dio ocasión para justificar, bajo la orientación del docente, la elección que el grupo realizó en cada oportunidad. De modo que la operatoria de la intervención del docente sobre este aspecto consistió en proponer que el grupo prediga qué compañero habrá resultado con mayor puntaje en los aspectos referidos a aspectos como: organización del estudio (preparación del lugar de estudio, asignación de tiempo, etc.), dedicación al estudio (interés y metas para estudiar, qué lugar ocupa en el día el estudio, cómo administrar las exigencias que éste conlleva), el esfuerzo invertido en el estudio (capacidad de resolver lo más dificultoso, nivel de atención que 
se dispensa frente a los distintos tipos de contenidos, etc.), aptitudes para el trabajo en grupo, etc. Luego de la elección del compañero o compañeros que más se acerquen al perfil en juego, se les pidió al grupo que justificaran la elección; es decir, este segundo paso consistió en detallar cómo las características implicadas en cada variable se notaban en el compañero elegido a la vista del resto de alumnos. En un tercer paso se le solicitó al compañero elegido que dé cuenta, afirmando, negando o ajustando, lo sostenido por sus compañeros. Esta experiencia resultó sumamente fructífera, por cuanto, además de crear clima propicio para reflexionar sobre las exigencias de una conducta académica exitosa contextualizada en relación con las problemáticas especificas del curso (determinados alumnos frente a determinados profesores), aportó información de gran valor para desarrollar las entrevistas tutoriales personales. Finalmente, cada sesión culminó, tras el debate orientado, con el ajuste y clarificación por parte del docente tendiendo a la apropiación por parte de los alumnos de mejores elementos para emprender la labor escolar.

Las entrevistas personales de tutoría pedagógica tuvieron una duración de entre 20 y 30 minutos. En ellas se refirieron aspectos surgidos en los encuentros grupales en el marco de los resultados personales del cuestionario MSLQ. En cada encuentro se le aclaró al alumno que los resultados del cuestionario manaban de su visión personal respecto del propio desempeño escolar y se lo invitó a revisar las ponderaciones, a justificarlas y finalmente a realizar los ajustes pertinentes.

Tanto en las intervenciones grupales como en las individuales, se diferenciaba el estudio fuera de clase (en el hogar) y el que se desarrollaba en el aula, en situación de clase; pues es evidente que una buena complementación de ambos escenarios resulta clave para la mejora del rendimiento escolar. Sobre esta última situación, la de "en el aula", se reflexionó sobre los modos de tomar apuntes, la clarificación de dudas, la advertencia de distractores para evitarlos, advertencia y administración de las preferencias personales por los distintos tipos de conocimiento propuestos desde la cátedra.

Con estas actividades se consiguió que el alumno se comprometiera de modo personal con la tarea escolar. El hecho de poner el estudio y sus exigencias como objeto de reflexión, permitió reconocer las conductas menos eficaces y, en gran medida, mejorarlas. A su vez, se provocó un mejor conocimiento de sí mismo en el alumnado, lo cual es base para el mejoramiento personal.

Como resultado de la intervención, un análisis de varianza mostró que en el rendimiento final hubo diferencias significativas $(\mathrm{F}=(1) 5.9, \mathrm{p}<.02)$ entre el grupo control y el grupo experimental, a favor de este último, mientras que en el comienzo del curso los grupos no se diferenciaban en este aspecto $(\mathrm{F}=(1) 2.55, \mathrm{p}=.11)$.

\section{CONCLUSIONES Y DISCUSIÓN}

En relación con las hipótesis planteadas, se ha observado que las medias más bajas se registran en la auto-regulación meta-cognitiva, la regulación del esfuerzo, el manejo del tiempo y del ambiente de estudio y las estrategias profundas de elaboración y de pensamiento crítico. Estas variables correlacionan significativa y positivamente con el rendimiento académico, teniendo más peso la regulación del esfuerzo.

Los resultados más relevantes son: 
1. La interpretación que los alumnos hacen de la auto-eficacia como una expectativa más que como una creencia en su capacidad de obtener buenos resultados.

2. El alto porcentaje de alumnos que muestran rasgos competitivos, poco deseables desde la visión de una educación integral, pero muy presentes en el medio social, lo que implica que en la acción pedagógica se debería insistir en el valor en sí del desarrollo personal y en destacar que el éxito social es una de sus consecuencias, que tiene valor en la medida en que refleja cualidades de excelencia.

3. Los resultados obtenidos respecto del valor que se da a la tarea que propone la escuela indican que se da a esta un valor meramente instrumental, lo que representa un desafío para la tarea docente, pues no se trata simplemente de señalar a los alumnos su valor en sí, sino de presentar los contenidos de modo que esta cualidad resulte evidente, lo que implica también un compromiso de valor del docente, del que surgirá espontáneamente su entusiasmo por lo que intenta transmitir.

4. Las diferencias por sexos en los patrones de atribución de éxito y de fracaso confirman lo observado en otras investigaciones respecto del predominio del sesgo defensivo en los varones y de la indefensión en las mujeres, a pesar de que esto contrasta con los resultados objetivos del rendimiento académico. El cambio de estos patrones exige que el docente asuma una tarea de formación en las virtudes morales, guiando al alumno en el auto-conocimiento y aceptación realista de las consecuencias de sus conductas y en los caminos para revertir los resultados no deseados.

5. Hay un déficit evidente en el empleo de estrategias cognitivas profundas, los alumnos se limitan a aplicar las que han aprendido en la escuela primaria. Sin duda, hay aquí una gran responsabilidad de la escuela y se hace necesario repensar la formación docente desde la perspectiva de la metodología de enseñanza.

6. También son deficitarias las estrategias de control de las conductas que aseguran un buen aprendizaje. En nuestro país es un rasgo preocupante la poca capacidad de esfuerzo de los adolescentes y jóvenes. Pensamos que sus causas son múltiples, pero entre ellas merecen señalarse la falta de presencia de valores de sentido de la vida, de metas vitales capaces de sostener es esfuerzo y la ejemplaridad negativa de un clima social que relativiza esas metas.

7. De las relaciones halladas entre el rendimiento académico y los estilos de aprendizaje, se podría inferir que los estilos de evaluación de los docentes favorecen la adopción de un estilo pragmático, porque los alumnos que regulan su esfuerzo de acuerdo con las exigencias de la escuela, con relativa independencia de la motivación intrínseca, son los que obtienen mejores calificaciones.

Sobre la base de estos resultados, se diseñó la intervención, basada fuertemente en la relación personal con los alumnos, a través de encuentros tutoriales, que tuvieron resultados positivos, tal como se observa a partir de las diferencias halladas entre el grupo control y el grupo experimental.

Es nuestra convicción que la formación de un alumno auto-regulado exige que el docente sea capaz de modelar conductas valiosas, de presentar metas de sentido y de asumir un compromiso con la formación integral, dentro de la que el rendimiento académico sea una consecuencia y no una meta última. 


\section{REFERENCIAS BIBLIOGRÁFICAS}

Bandura, A. (1977). Self-efficacy: toward a unifying theory of behavioural change. Psychological Review, vol. 84, n. 2, 191-215.

Bandura, A. (1993). Perceived self-efficacy in cognitive development and functioning. Educational Psychologist, vol. 28, n. 2, 117-148.

Bandura, A. (1995). Exercise of personal and collective efficacy. En A. Bandura (Ed.), Self-efficacy in Changing Societies (pp. 1-45). California: University of Cambridge Press.

Bandura, A. (1997). Self-efficacy: The exercise of control. New York: Freeman.

Boekaerts, M. y Niemivirta, M. (2000). Self-regulated learning. Finding balance between learning goals and ego-protective goals. En M. Boekaerts, P. Pintrich (Eds.), Handbook of self-regulation (Cap. 13). San Diego, CA: Academic Press.

Boekaerts, M. y Corno, L. (2005). Self-Regulation in the Classroom: A Perspective on Assessment and Intervention. Applied Psychology: An International Review, vol. 54, n. 2, 199-231.

Eccles, J. (1983). Expectancies, values and academic behaviours. En J. Spence (Ed.), Perspective on achievement and achievement motivation (pp. 75-146). San Francisco: Freeman.

Kuhl, J. (1984). Volitional aspects of achievement motivation and learned helplessness: toward a comprehensive theory of action control. En Progress in experimental personality research, Vol. 13 (pp. 99-171). Orlando: Academic Press.

Kuhl, J. (1985). Volitional mediators of cognition-behaviour consistency: Self-regulatory processes and action versus state orientation. En J. Kuhl, y J. Beckman (Eds.), Action control: from cognition to behaviour (pp. 101-128). Berlin: Springer-Verlag.

Martin, A. J., Marsh, H.W. y Debus, R.L. (2001a). A quadripolar need achievement representation of self-handicapping and defensive pessimism. American Educational Research Journal, vol. 38, n. 3, 583-610.

Martin, A. J., Marsh, H.W., y Debus, R.L. (2001b). Self-handicapping and defensive pessimism: Exploring a model of predictors and outcomes from a self-protection perspective. Journal of Educational Psychology, vol. 93, n. 1, 87-102.

Pintrich, P. (2000). The role of goal orientation in self-regulated learning. En M. Boekaerts, P. Pintrich y M. Zeidner (Eds.), Handbook of self-regulation (cap. 14). San Diego. CA: Academic Press.

Pintrich, P. y De Groot, E. V. (1990). Motivated and self-regulated learning components of classroom academic performance. Journal of Educational Psychology, vol. 82, n. 1, 33-40.

Pintrich, P. y Zusho, A. (2002). The development of academic self-regulation: The role of cognitive and motivational factors. En A. Wigfield y J.S. Eccles (Eds.), Development of achievement motivation (pp. 249-284). San Diego, CA: Academic Press.

Puustinen, M. y Pulkkinen, S. (2001). Models of Self-regulated Learning: a review. Scandinavian Journal of Educational Research, vol. 45, n. 3, 269-286.

Schunk, D. H. (2005). Self-Regulated Learning: The Educational Legacy of Paul R. Pintrich. Educational Psychologist, vol. 40, n. 2, 85-94.

Seifert, T. L. (1997). Academic goals and emotions: results of a structural equation model and cluster analysis. British Journal of Educational Psychology, vol. 67, n. 3, 323-338.

Seifert, T. L. (2004) Understanding student motivation. Educational Research, vol. 46, n. 2, 137-149.

Zimmerman, B. J. (2000). Attaining Self-Regulation: A Social Cognitive Perspective. En M. Boekaerts, P. Pintrich y M. Zeidner (Eds.), Self-Regulation: Theory, Research and Application (pp. 13-39). Orlando, FL: Academic Press.

Zimmerman, B. J. (2002). Becoming a Self-Regulated Learner: An Overview. Theory Into Practice, vol. 41, n. 2, 64-71. 